Accepted by The Astronomical Journal

\title{
Optimal proper motion measurements with the Wide Field and Planetary Camera
}

\author{
Rodrigo A. Ibata ${ }^{1}$, Geraint F. Lewis ${ }^{2}$
}

\begin{abstract}
An optimal maximum-likelihood technique for computing point-source image centroids from many, slightly offset, CCD frames is presented. The method is especially useful for measuring stellar proper motions from data taken with the Wide Field and Planetary Camera aboard the HST, and also provides a means to identify very compact non-stellar sources. We work though the example problem of obtaining image centroids of objects in the Hubble Deep Field.
\end{abstract}

Subject headings: astrometry — stars: kinematics — Galaxy: kinematics and dynamics

\section{Introduction}

The Wide Field and Planetary Camera (WFPC2) aboard the Hubble Space Telescope (HST), is an excellent tool for astrometric study. This is a result of its superb spatial resolution and image stability. However, the Wide Field (WF) camera produces undersampled images, and the point spread function (PSF) of WFPC2 is complex. These properties force one to have to take special care in the data reduction process.

In the present contribution we discuss an optimal maximum-likelihood technique that is particularly well-suited for calculating centroids of point-like objects from WFPC2 data

\footnotetext{
${ }^{1}$ European Southern Observatory Karl Schwarzschild Straße 2, D-85748 Garching bei München, Germany Electronic mail: ribata@eso.org

${ }^{2}$ Fellow of the Pacific Institute for Mathematical Sciences 1998-1999, Dept. of Physics and Astronomy, University of Victoria, Victoria, B.C., Canada \& Astronomy Dept., University of Washington, Seattle WA, U.S.A.

Electronic mail: gf1@uvastro.phys.uvic.ca

Electronic mail: gfl@astro.washington.edu
} 
obtained in many slightly offset exposures. The same technique will be readily applicable to STIS imaging data or Advanced Camera images.

As a working example, we present the problem of obtaining image centroids for point-like objects in the Hubble Deep Field (HDF).

\section{Astrometry with WFPC2}

The expected proper motion (PM) $\alpha$ for a point-source at distance $d$, moving with transverse velocity of $v_{\perp}$ over a time interval of $T$ is $\alpha=1.79 \frac{v_{\perp}}{200 \mathrm{~km} \mathrm{~s}^{-1}} \frac{T}{2 \mathrm{yr}}\left(\frac{d}{1 \mathrm{kpc}}\right)^{-1}$ pixels on the Planetary Camera (PC) and $\alpha=0.82 \frac{v_{\perp}}{200 \mathrm{~km} \mathrm{~s}^{-1}} \frac{T}{2 \mathrm{yr}}\left(\frac{d}{1 \mathrm{kpc}}\right)^{-1}$ pixels on the WF camera (the velocity, distance and time interval values are appropriate to our test problem below). So the HST guiding accuracy of 0.005 arcsec RMS (corresponding to 0.1 PC pixels and 0.05 WF pixels) in "fine lock" mode, is sufficient to measure the PMs of Galactic stars out to large distances, as long as accurate image centroids and a suitable reference frame can be determined to better accuracy than the expected proper motions. This jitter of 0.005 arcsec, will make the undersampled WFPC2 images fractionally wider, but it will not significantly affect image centroiding (to better than the RMS uncertainty), unless the jitter has a systematic direction.

The WFPC2 dithering technique, where many exposures of a field are taken with slightly different pointings, offset by a few pixels, was developed as a means to eliminate the effects of CCD cosmetic defects, hot pixels and cosmic rays. It is also very useful for astrometric purposes, since the positional accuracy of each dithered frame allows the stellar image, which is undersampled in a single frame, to be resampled at several sub-pixel positions. Specialized software, such as the "drizzle" algorithm of Fruchter \& Hook (1997), have been devised to stack dithered frames. Taking advantage of the extra positional information, they dramatically enhance the resolution of the final stacked image.

However, stacking inevitably degrades information. The resulting PSF must always be more complex than that of individual frames, and given that the WPFC2 PSF varies strongly as a function of position over the camera, the PSF of the stacked image will also depend on the particular dither pattern adopted by the observer. These problems become more severe if one enhances the resolution of the stacked image (with such algorithms as "drizzle"); furthermore, the noise in the stacked image will then be spatially correlated and hence quite complex. On the other hand, working purely with shallow individual frames is a huge waste of the depth of the data-set (in the HDF, objects that have $\mathrm{S} / \mathrm{N} \sim 8$ in the combined 58-exposure first epoch F814W stack, correspond to $\sim 1 \sigma$ detections in individual 
exposures).

The solution to this apparent dilemma is very simple. We assume the PSF in each frame (and its variation across each frame) is known. These PSFs are best determined from the data-set under investigation. [3 However, if there are few or no bright, isolated, unsaturated stars present in those frames, one may still be able to obtain a good approximation to the PSF, if it sufficiently stable over time. This is the case for WFPC2; and suitable data are readily obtained from the archive. We also assume that the transformations (and inverse transformations) that map every point on the $i$ th frame to a Cartesian grid on the sky have been determined in advance (using techniques such as those described below). A "master frame", whose rows and columns are aligned with that Cartesian grid is produced by stacking all the individual frames (using the "drizzle" algorithm, for instance, but with resolution enhancement turned off). On this "master frame", we search for candidate stars and photometer them; a crowded-field photometry package such as "ALLSTAR" (Stetson $1994)$ is ideal for this purpose. This also yields a first estimate $\left(x_{j}, y_{j}\right)$ of the astrometric position on the "master frame" of the $j$ th candidate point-source. These positions are transformed into the coordinate system of the $i$ th frame to give $\left(x_{j}^{i}, y_{j}^{i}\right)$. Then, for each of the $j=1, \ldots, N$ stellar candidates, we find the likelihood of the the exposure-normalized PSF model in the $i$ th frame, given the data $D^{i}$ in the $i$ th frame

$$
L_{j}^{i}\left(x_{j}^{i}, y_{j}^{i}\right)=\prod_{k, l} L\left[\operatorname{PSF}^{i}(k, l)+S_{j}^{i} \mid D^{i}(k, l)\right],
$$

where the product is performed over all uncontaminated pixels $k, l$ within a circle of radius $R$ of $\left(x_{j}^{i}, y_{j}^{i}\right)$. Contaminated pixels - by which we mean pixels on cosmetic defects, hot pixels, or pixels affected by cosmic ray impacts - are simply left out of the calculation (we discuss below how bad pixel maps were constructed for each frame of our test data-set). The quantity $S_{j}^{i}$ is the modal sky value in an annulus with suitably chosen inner and outer radii around the $j$ th object on frame $i$. The product over all $M$ frames of $L_{j}^{i}$,

$$
L_{j}\left(x_{j}, y_{j}\right)=\prod_{i=1, \ldots, M} L^{i}\left(x_{j}^{i}, y_{j}^{i}\right)
$$

is the likelihood that a point-source is centered at position $\left(x_{j}, y_{j}\right)$ on the "master frame", given all the available data. Repeating this process in a fine grid of $(x, y)$ values in the immediate neighborhood of $\left(x_{j}, y_{j}\right)$, yields the likelihood surface, and a two-dimensional maximization routine can then be used to find the position $\left(X_{j}, Y_{j}\right)$ of the maximum of $L_{j}$. The coordinate $\left(X_{j}, Y_{j}\right)$ is then the most likely position of the center of the image. There

\footnotetext{
${ }^{3}$ If there is a systematic direction to the telescope jitter which changes from exposure to exposure, its effect can be largely eliminated by measuring the PSF of each data-frame individually.
} 
has been no image degradation, as the data have not been tampered with (except for initial debiassing and flat-fielding), and there is no loss of depth. The technique is optimal, and is especially useful for the case where rotated, optically distorted frames with different and (or) spatially varying PSFs are to be analyzed.

The important noise sources are: Poisson noise in the source, Poisson noise in the sky, read noise, flat-fielding errors and PSF mismatching errors. When dealing with faint objects on a low sky background it will be advantageous to consider carefully the distribution of expected counts, which is why we stressed the use of the likelihood function above. However, for all the images we analyzed, the sky background was substantial, greater than $100 \mathrm{e}^{-}$, so that the noise distribution could be reasonably modeled by a Gaussian error distribution on each pixel. Given this, one may then trivially compute, using the $\chi^{2}$ statistic instead of the likelihood in the computations above, the probability that the observed brightness enhancement is drawn from the same distribution as a point-source located at $\left(X_{j}, Y_{j}\right)$. Thus, this method also provides an excellent means of discriminating point-like from extended sources, that is especially powerful at revealing objects that deviate only slightly from point-sources. Again, this uses the full depth of the data-set, avoids the information degradation inherent to the stacking process, and also avoids the problems of having correlated noise.

We found that a considerable improvement in $\chi^{2}$ can be achieved if the magnitude as well as the position of bright candidate point-sources are refined simultaneously. Though the flux estimate did not vary from the input value by more than 0.1 magnitudes for any of the objects we measured in the test problem below, the $\chi^{2}$ probability occasionally improved by orders of magnitude.

Although we have not tested this technique on crowded fields, it is likely to yield accurate centroid positions if the input positions and magnitudes of all detectable stars have been carefully determined with a good crowded-field photometry package. However, one should make the following two alterations to the algorithm. First, before analyzing the $j$ th object, one should subtract the expected counts from all other objects from each frame in the data set. And second, the objects should preferably be analyzed in order of decreasing flux.

A limitation of our method is that we choose not to measure variability over the time-span of the observations in any one epoch. Clearly, adding an extra parameter for each object on each frame, would make the scheme less robust. Variability between epochs can be measured, however. 


\section{An example: Proper motions in the Hubble Deep Field}

The HDF is the deepest image yet obtained of the Universe; taken with the Hubble Space Telescope (HST) in director's discretionary time in December 1995 (Williams et al. 1996), its primary aim of studying the formation an evolution of galaxies has been extremely successful.

Apart from the numerous galaxies, a small number of stars were also detected in this field. However, the constraints that can be placed upon Galactic structure models from these data are disappointing due to the relatively shallow limit of $\mathrm{I}=26$ at which stars can be discriminated from the galaxies with reasonable confidence (Flynn et al. 1996). For comparison, the limiting magnitude of the HDF in F814W is I $\sim 28$, so a factor of $\sim 15$ of survey volume would be gained if one could push star-galaxy discrimination to the faintest limit of the data.

Here we show that a better way to find stars at the faint limit of this data set is to observe the field in $\mathrm{F} 814 \mathrm{~W}$ in a second epoch, and calculate proper motions (PMs). The necessary follow-up data, taken in December 1997, were obtained to undertake a search for high redshift supernovae (Gilliland \& Phillips 1998). Note that over the two year timespan that separates the datasets, a star $1 \mathrm{kpc}$ away travelling with a transverse velocity of $200 \mathrm{~km} \mathrm{~s}^{-1}$ will move $0.82 \mathrm{WF}$ pixels. Transverse velocities of this magnitude can be expected from spheroid (or more interestingly) halo stars due to the large velocity dispersions of these populations. Furthermore, their slow rotation about the Galactic center will introduce a large apparent motion as viewed from the Sun.

\subsection{Registration of frames}

To apply the method outlined above, we need to know the geometrical transformations between pixel positions on each frame taken at a given epoch. To do this, we could find the centroids of objects (stars or galaxies) on each frame and compute transformation coefficients. However, due to optical design, WFPC2 images give a substantially distorted view of the sky, so a high order polynomial must be used to give acceptable residuals. If there are few objects on the frames for which reliable centroids can be found, this procedure will be far from robust. It is better therefore to make use of some prior information: the optical distortion of WFPC2, as a function of wavelength, is fairly well understood. Trauger et al. (1995) have published transformations (a 10-coefficient bicubic polynomial in each of the $x$ and $y$ directions) that allow one to convert CCD pixel positions, in a given passband, to a geometrically corrected frame. The accuracy of this transformation has been 
determined to be 0.1 pixels RMS over the fields of view of the CCDs.

Though not necessary, the proper motion analysis is easier if one is able to construct an accurate extragalactic reference frame. Ideally, one would like to use point-like sources for this purpose, as the centroid of an extended source (which is most probably also lumpy) is not easily defined. However, to date no QSOs have been identified in the HDF. One is therefore forced to use galaxies to define the reference frame.

Galaxies are generally fuzzy, lumpy objects, so it is very difficult, if not perhaps impossible, to define the center of the light distribution. To circumvent this problem, the approach we take is to obtain differential measurements. Only for the purpose of determining the transformations between frames, we "un-distort" all of the frames by applying the Trauger et al. (1995) coefficients. Bad pixels in the frames are flagged, as described below. The dither position \#6 frames (see Williams et al. 1996) were chosen to define a "reference frame". A first estimate of the positions of the $\sim 50$ brightest galaxies in the chosen "reference frame" is obtained using the "FIND" algorithm of "DAOPHOT" (Stetson 1987), which fits a Gaussian function to brightness enhancements in the image. However, the resulting positions are accurate to not much better than about 1 pixel. To improve this positional accuracy, we implemented the following algorithm.

First, a search is performed for the local minima of the marginal density distributions along the column and row directions of a 40 pixel box centered on each input position. This is done in two iterations following the first steps of the recipe given in Stetson (1979). These local minima are found on either side of the peak, both in in the $x$ and $y$ directions, and are used to define the limits of a new box surrounding the object under study. This box will be free of the influence of brighter neighbors. The marginal density distributions in this new box are computed. The algorithm then cross-correlates the marginal density distributions of the same object on each frame. Obviously, best results will be obtained if the frames being compared have approximately the same orientation angle. 7

Having determined the positional offsets for all the bright galaxies on all frames, one can proceed to find the geometric transformations that relate the frames to each other. A simple four-coefficient geometric transformation (shift, rotation and change of scale) was found to give excellent residuals, better than 0.02 pixels RMS, between frames in the same passband. Thus the Trauger et al. model provides an accurate map of the WFPC2 optical distortion. (The fact that we are able to align the undistorted frames to better than

\footnotetext{
${ }^{4}$ We also experimented performing two-dimensional cross-correlation of the cut-out images. The accuracy achieved is approximately the same as in the procedure outlined above, but less robust, in that wildly wrong answers were often returned when the images did not have high signal to noise.
} 
the expected accuracy of the distortion transformations is probably a consequence of the relatively close alignment of the individual HDF frames).

This procedure has given us the geometric transformations between the (undistorted) "reference frame" and all other undistorted frames. However, what we really need to know are the transformations (and inverse transformations) between the raw (optically distorted) frames and the "reference frame". The forward transformations are found by simply substituting the Trauger et al. functions into the above four-coefficient geometric transformations. The inverse transformations cannot be written down as a polynomial, but a non-linear Newton-Raphson algorithm can be used to provide the required inverse mapping.

\subsection{Bad pixel rejection}

Finally, it is necessary to flag bad pixels on the raw frames. Consider pixel $k, l$ on the $i$ th frame. We find the overlap area (using the computed geometrical transformations) of the footprint of this pixel on all other frames in the same passband as frame $i$ and in the same epoch, to obtain a list of $M$ flux estimates (i.e. counts per second above the sky) at this position. The mean and standard deviation of this list are computed, after clipping the highest flux datum. Given the exposure lengths and the number of frames, this datum is likely to be severely contaminated by a cosmic ray; however, the statistical bias introduced by this clip should be negligible. The pixel is flagged as bad if the measured flux deviates from the mean flux by more than four standard deviations. This process is repeated for all pixels on all frames. The advantage of this procedure is clear: maximum spatial resolution is maintained on all frames.

\subsection{Sample results}

As an illustration, in Figure 1 we show the result of applying the present technique to three sample objects on the WF2 chip using data from the first epoch HDF. The upper two panels show results relating to a fairly faint star identified by Flynn et al. (1996) (I=24.78, $\mathrm{V}-\mathrm{I}=1.64$; position on HDF WF2 dither $\# 6: x=350, y=469$ ). Panel (a) shows the likelihood contours of the centroid of this star (in the "reference frame"), calculated using all first epoch F814W exposures. The "star" graph-marker shows the point $\left(X_{j}, Y_{j}\right)$, the most likely centroid position, while the $n$th contour marks the boundary of the region

where the likelihood has fallen by a factor of $\exp -\frac{n^{2}}{2}$ from the most likely value (so the 
image centroid is $<10^{-22}$ times less likely to be situated beyond the last contour than at the position of the "star" marker). The distance from the "star" marker to the first contour is 0.017 pixels, or 1.7 mas. Panel (b) displays the object profile. The position $\left(X_{j}, Y_{j}\right)$ is transformed to the correct position on the individual raw frames, $\left(X_{j}^{i}, Y_{j}^{i}\right)$; for all frames $i$, we plot the raw pixel data within 2 pixels of $\left(X_{j}^{i}, Y_{j}^{i}\right)$ as a function of distance from that point. The uncertainties on individual pixel values are also indicated. The expected counts from the PSF models are shown as filled circles; that these values do not always decrease monotonically from the image center is due to non-axisymmetry in the model PSFs, and to the particular way in which the dithering sampled the object. This diagram serves simply

to illustrate the goodness of fit of the data to the PSF; we find that the probability that $\chi^{2}$, for a correct model, should be less than the observed value is $P=0.47$.

The middle two panels display the results of applying the technique to a faint, blue star identified by Flynn et al. (1996) ( $\mathrm{I}=26.22, \mathrm{~V}-\mathrm{I}=0.16$; position on HDF WF2 dither \#6: $x=322, y=637$ ). Panels (b) and (c) have, respectively, similar content to panels (a) and (b). Here, the centering uncertainty has degraded to $\sim 3$ mas. Using the $\chi^{2}$ statistic, we find $P=1.9 \times 10^{-4}$, so this object is almost certainly not a point-source, illustrating the resolving power of our method. Interestingly, other faint blue objects identified by Flynn et al. (1996) as stars can similarly be shown to be non-stellar; the nature of these objects will be investigated in a subsequent contribution.

Finally, panels (d) and (e) show the results for an object at the limit of the HDF, $(\mathrm{I}=27.9$, no color information available; position on HDF WF2 dither \#6: $x=326$, $y=250$ ). Our estimated centroiding accuracy on this extremely faint object is $\sim 10$ mas.

\subsection{Positional accuracy}

Many sources contribute to uncertainty in the computed centroid positions. There are "fundamental" uncertainties from Poisson noise in the sky and in the object, from detector noise, and from the sampling. There will also be uncertainties in the flat-fielding, and in the PSF determination. Further sources of uncertainty, not accounted for in our model, arise from the fact that CCD pixels are not exactly square, that they are not laid out on a perfect Cartesian grid, and that, at some level, every pixel has a non-uniform sensitivity over its surface.

Clearly, it is desirable to determine the combined effect of all these uncertainties. To this end, we separated the first epoch F814W data at dither positions 1-5 and at 
dither positions 6-11 to make two sub-samples. Comparing the positions of point-sources determined from the first sub-sample $\left(\overrightarrow{x_{1}}\right.$, say) to those determined in the other $\left(\overrightarrow{x_{2}}\right)$, provides an internal means to measure the accuracy of the method. The HDF frames are slightly shifted and some are slightly rotated with respect to each other, so this exercise should provide a good indication of the achievable centering (and proper motion) accuracy for a dataset where the frames of all epochs are in close alignment.

This experiment was performed on the seventy-two objects, with light profiles consistent with being point-sources with probability $P>0.01$, that we detected in the HDF. The results are displayed in Figure 2. The "star" graph-markers show the value of $\frac{\left|\overrightarrow{x_{1}}-\overrightarrow{x_{2}}\right|}{\sqrt{2}}$ as a function of I magnitude. The filled circles give the expected uncertainties, as derived from the likelihood surfaces. The good agreement between these two methods of estimating the centroiding uncertainties suggests that our noise model is reasonable. Clearly, it is possible to obtain quite accurate centroids (to $\sim 10$ mas) even at the very faint limit of the HDF, opening the possibility of many interesting studies.

\section{Conclusions}

A method has been outlined for obtaining accurate point-source image centroids from WFPC2 data. It is optimal, in the sense that maximum-likelihood techniques are used to take advantage of all available positional and brightness data contained in the CCD frames.

It is shown that, when applied to the HDF data-set, centroid uncertainties on the order of 2 mas are easily achieved for relatively faint stars, while stars at the limit of the data-set, near $\mathrm{I} \sim 28$, may be measured with accuracies of $\sim 10$ mas. Many interesting proper motion studies are therefore possible.

Further work is required to determine up to what accuracy the bulk proper motion of point-sources ( e.g., star cluster or local group galaxies) improves as the square root of the number of sources. Systematic effects must drown the signal at some level; but judging from the present work, our 0.02 pixel limit is set by the accuracy with which we were able to fix the reference frame. The bulk proper motions of even very faint populations can therefore be measured down to at least that level of accuracy. So for projects that require proper motion measurements of very faint sources, HST imaging instruments are likely to remain highly competitive even compared to the next generation of astrometric missions such as NASA's Space Interferometry Mission (SIM) or ESA's Global Astrometric Interferometer

\footnotetext{
${ }^{5}$ data was not obtained at all dither positions in all filters
} 
for Astrophysics (GAIA).

The proper motions measured between the original HDF and the second epoch exposures in that field will be analyzed and presented in a forthcoming paper (Ibata et al. 1998).

\section{REFERENCES}

Gilliland, R., Phillips, M. 1998, in prep

Flynn, C., Gould, A., Bahcall, J. 1996, ApJ 466, L55

Fruchter, A., Hook, R. 1997, in Applications of Digital Image Processing XX, ed. A. Tescher, Proc. S.P.I.E. vol. 3164

Ibata, R., Gilliland, R., Richer, H., Scott, D. 1998, in prep

Stetson, P.B., 1994, PASP 106, 250

Stetson, P.B., 1987, PASP 99, 613

Stetson, P.B., 1979, AJ 84, 1056

Trauger, J., Vaughan, A., Evans, R., Moody, D. 1995, in Calibrating the Hubble Space Telescope: Post Servicing Mission, edited by A. Koratkar and C. Leitherer (STScI, Baltimore), p. 379

Williams, R. et al. 1996, AJ 112, 1335 


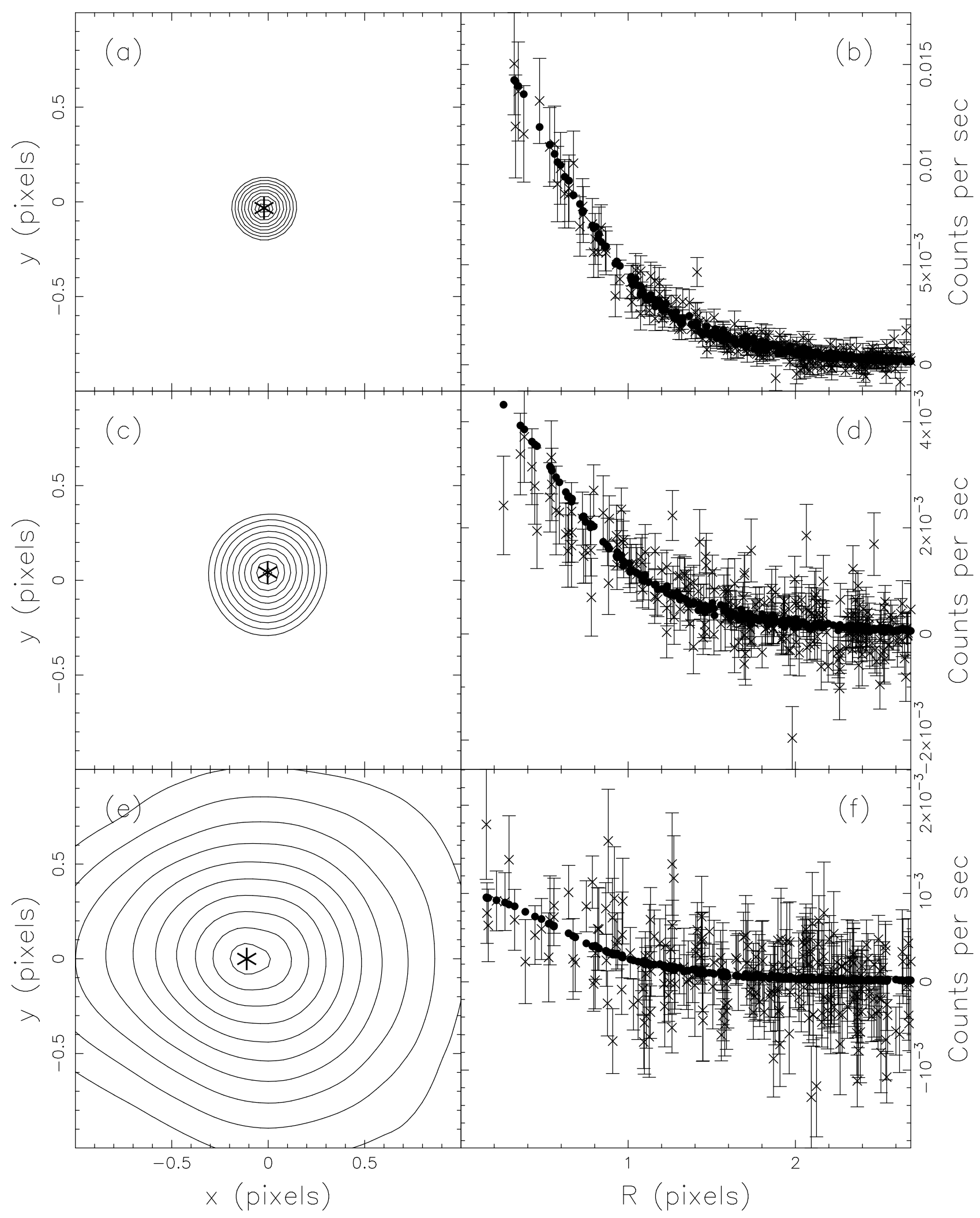

Fig. 1. - The centroid likelihood surfaces and image profiles of three stars of magnitude $\mathrm{I}=24.78, \mathrm{I}=26.22$, and $\mathrm{I}=27.9$ are displayed, respectively, in the upper, middle and lower panels. A detailed explanation of these diagrams is given in the text. 


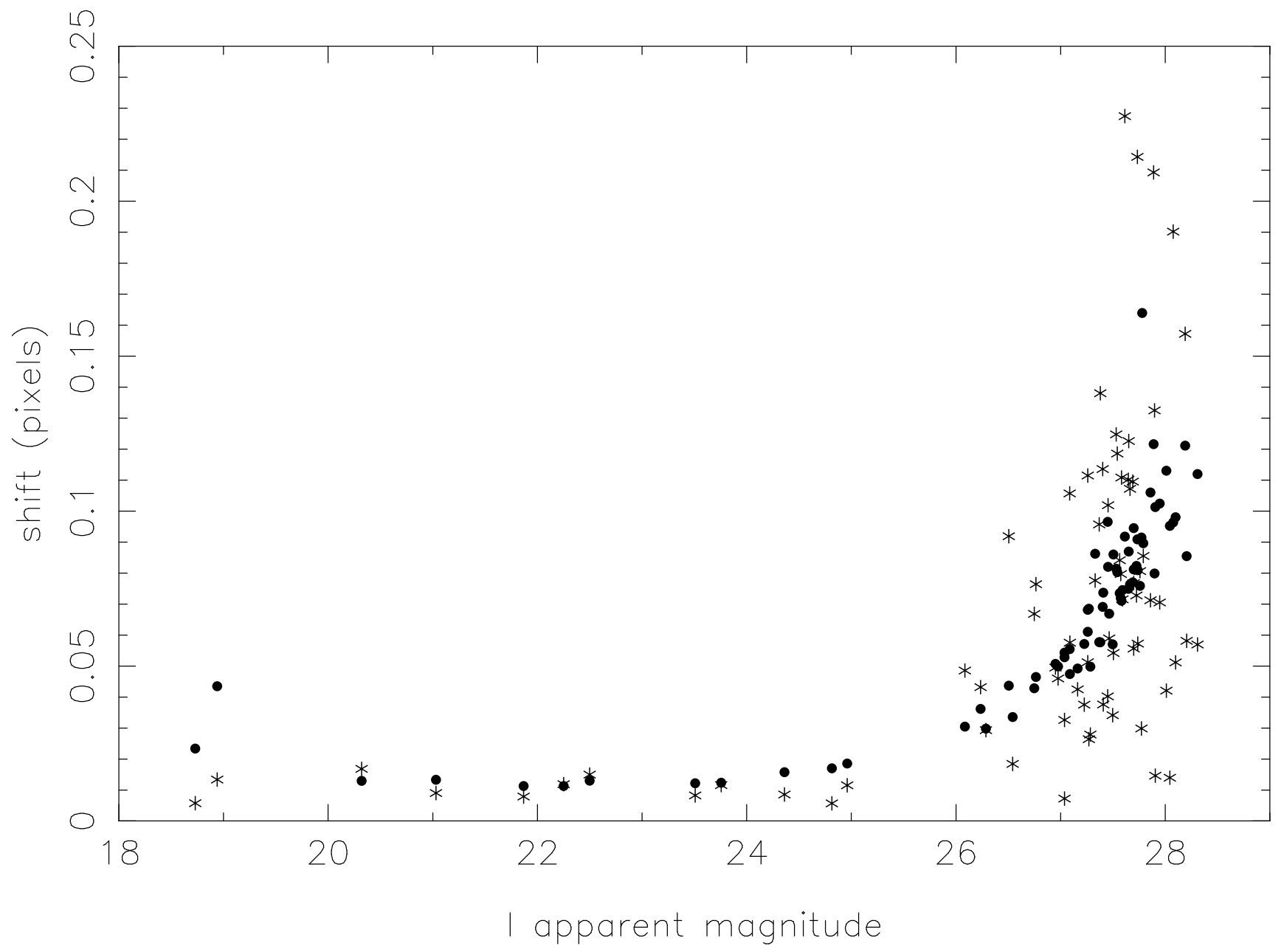

Fig. 2.- The uncertainty in the centroid position of point-like objects on the HDF WF chips is displayed as a function of I-band apparent magnitude. The "star" markers show $\frac{\left|\overrightarrow{x_{1}}-\overrightarrow{x_{2}}\right|}{\sqrt{2}}$, that is, $1 / \sqrt{2}$ times the difference in the centroid position derived from two, approximately equal exposure, subsamples of the original HDF dataset. The filled-circles show the expected uncertainties, derived from the centroid likelihood surfaces. 